\title{
NC NODE SELECTION GAME IN COLLABORATIVE STREAMING SYSTEMS
}

\author{
Nikolaos Thomos, Hyunggon Park, Eymen Kurdoglu and Pascal Frossard \\ Signal Processing Laboratory (LTS4) \\ Swiss Federal Institute of Technology, Lausanne (EPFL) \\ \{nikolaos.thomos, hyunggon.park, eymen.kurdoglu, pascal.frossard\}@epfl.ch
}

\begin{abstract}
Network coding has been recently proposed as an efficient method to improve throughput, minimize delays and remove the need for reconciliation between network nodes in distributed streaming systems. It permits to take advantage of the path and node diversity in the network when the network coding nodes are placed efficiently. In this paper, we investigate networks consisting of nodes that autonomously determine whether they should perform network coding or not as well as their set of parent nodes. Each node makes its decisions that maximize its quality of service. The decisions include the selection of operation mode (i.e., network coding mode, simple data forwarding mode) and the selection of extra connections. The resulting interactions among the nodes are modeled as a congestion game, thereby ensuring an equilibrium, i.e., stable multimedia stream flow. The experimental results show that the proposed scheme is appropriate for distributed multimedia transmission since it provides a stable quality without imposing centralized control.
\end{abstract}

Index Terms - Randomized network coding, congestion game, distributed multimedia streaming.

\section{INTRODUCTION}

The recent development of low-cost hardware devices has enabled the wide deployment of overlay mesh networks such as sensor networks. These networks consist of nodes that can self-organize into groups, or can join or leave the network with no notification. These dynamics often result in bottlenecks that degrade the quality of the transmitted content which is critical in delay sensitive applications such as multimedia streaming. In such networks, the synergistic actions of nodes participating in the transmission are desirable and permit them to improve the quality of the transmitted content. However, nodes are usually self-interested entities that would like to maximize their own utility. Thus, these interactions among nodes can be modeled as a game [1], where each node can cooperate with or compete against the others by determining its own actions in order to improve its quality of service.

The considered overlay mesh networks are characterized by significant path diversity. Thus, nodes receive information through various paths. However, several links can be significantly congested due to excessive number of users simultaneously access them. To efficiently utilize the shared links, network coding [2] has been adopted by recent streaming systems [3-5]. Unlike conventional data transmission systems simply forwarding packets, the network coding schemes send combinations of the received packets. The network coding operations are performed in a large Galois field [4] to ensure that the probability of regenerating a packet is negligible.

This work was supported by the Swiss National Science Foundation grants 200021-118230 and PZ00P2-121906.
Early network coding systems determine the coding coefficients. This is infeasible and inefficient for large scale networks with heterogeneous users as significant bandwidth should be wasted for communicating topological data and network statistics. They also assume a central node that determines the coding coefficients and coordinates the delivery. Randomized network coding $(\mathrm{RNC})^{1}[6]$ is more appropriate for such ad-hoc networks since RNC systems do not impose coordination between nodes for determining the coefficients. Motivated by the success of RNC, a practical network coding system has been proposed in [4] that addresses most of practical issues that prohibit RNC application to real systems. Specifically, it devises a special protocol to deal with buffering issues and timing constraints. It also introduces the concept of generations by restricting the coding operations on packets sharing similar decoding deadlines. The network coding coefficients are embedded into data packets for providing to clients the information required for recovering the data by Gaussian elimination. The clients are able to decode upon collection of a full rank system of packets. To avoid transmission of redundant packets, the nodes check whether the received ones are innovative (i.e., the packets that contain novel information) and discard noninnovative packets.

In practice, it is however not ideal to require that every node performs network coding. This leads to large delays, increasing complexity and even lower decoding probability in large scale networks, since clients are often not able to receive a full rank system of packets before the packets expire. In this paper, we propose a distributed solution for the placement of network coding nodes, such that the quality of service is improved. The problem of positioning the network processing nodes is similar in some concepts to the work in [7] where few network-embedded FEC nodes (NEF) are placed in networks organized in multicast trees. In this work, the nodes first decode the video data upon reception of a sufficient large number of packets and successively re-encode it. The optimal placement is found by a greedy algorithm that requires extensive computational cost. We address here a more general problem and we propose a distributed solution for overlay networks that cannot be trivially organized into multicast trees. We design a protocol that provides incentives for nodes to perform RNC, as it increases its individual performance, but it can also improve the system performance. The nodes that turn into RNC nodes are rewarded by extra connections with other nodes that can supply them with additional innovative rate. RNC nodes can change their parents if they cannot acquire the desired multimedia quality. When a node is connected to another parent node, the quality improvement can be estimated based on the information about the expected bandwidth allocation from the parent node. The interactions are modeled as a congestion game, and thus, an equilibrium for nodes' actions is always guaranteed. The proposed method is general and appropriate for transmitting any type of

\footnotetext{
${ }^{1}$ Throughout the paper the terms RNC and NC are used interchangeably.
} 
data. The experimental evaluation makes clear that utility fair solution are guaranteed by devising a protocol that allows decentralized decisions.

\section{PROBLEM DESCRIPTION}

We investigate multimedia streaming in overlay mesh networks that can be modeled as directed acyclic graphs. The graphs are denoted by a pair $G=(V, E)$ where $V$ is the set of network nodes and $E$ the set of edges (links) in the network. The overall network comprise $N$ network nodes of three types: $N_{s}$ servers, $N_{i}$ intermediate nodes and $N_{c}$ clients, i.e., $N_{s}+N_{i}+N_{c}=N$. Thus, $V=\left\{V_{s}, V_{i}, V_{c}\right\}$ is the union of sets $V_{s}, V_{i}$ and $V_{c}$ which are respectively the set of servers, intermediate nodes and client nodes.

We assume that the servers stream the same multimedia sequence, possibly with different quality layers, to the system users. All the nodes in the system (except the servers) are interested by decoding the multimedia streams. The intermediate nodes $j \in V_{i}$ can be NC nodes, which perform RNC with the received packets, or simply relay nodes $\overline{N C}$, where a "store and forward" approach is followed. $\overline{N C}$ nodes perform random replication to fill the outgoing links whenever their cumulative incoming rate $R_{j}^{I}$ is smaller than their outgoing rate $R_{j}^{O}$. All servers are $N C$ for improved system's robustness to packet erasures and network dynamics as through RNC the packet diversity is maximized and the importance of the transmitted packets is flattened as packet duplicates are eliminated and all packets contribute equally to the data quality and useful only when a sufficient number of packets is collected by the clients. Large Galois fields $\operatorname{GF}\left(2^{q}\right)$ are employed to reduce the probability of receiving packet replicas from multiple sources as well as the probabilities of generating duplicate packets due to independent coding processes.

In order to deal with heterogeneous receivers and different access bandwidths, we consider scalable multimedia streams that are able to offer different quality of service $Q_{i}$ depending on the number of layers that are correctly decoded. The network coding nodes therefore implement an embedded RNC solution [8] that provides unequal importance protection to the set of packets. This permits to take advantage of the unequal importance of the layered data and allow nodes with more resources (higher cumulative download rate) to reconstruct higher quality multimedia streams, while nodes with fewer resources to be able to decode a basic quality multimedia stream. Therefore, the more important data is stronger protected and can be decoded by the majority of the nodes. We assume that the packets are divided into $L$ quality layers with the first layer containing the most important information, while the $L^{\text {th }}$ layer stands for the least important. In particular, we define as class $i$ the set of packets that are linear random combinations of native (unencoded) packets from the first $i$ layers. In practice, the class of a packet is identified by a small header which is appended to each packet.

We are interested in the problem of determining which intermediate nodes in the system should perform network coding, in order to improve their own quality of service. In large networks, the delay, the complexity and even the decoding failure probability generally increase when network coding is performed in all the nodes. We propose a distributed solution where nodes can decide autonomously to turn into a network coding mode. As an incentive, the nodes that perform network coding are allowed to request an extra link for acquiring more download bandwidth, which increases the incoming innovative rate, hence the media quality. Whenever a node $j$ becomes $N C$ node it cancels out the replication effect of its $\overline{N C}$ parent nodes as it is very unusual that a packet combination is regenerated multi-

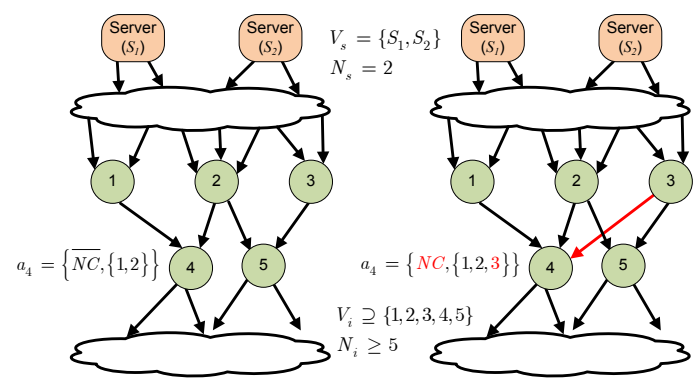

Fig. 1. Proposed rate allocation game. Original rate allocation (left). Rate allocation after the node $n_{4}$ becomes an RNC node (right).

ple times. In fact, RNC nodes act similarly to sources in the sense that they refresh the set of packets in the network by eliminating the packet duplicates. In the next section, we present a new protocol that permits to select the network coding nodes.

\section{PROPOSED PROTOCOL DESIGN AND ANALYSIS}

In this section, we describe the proposed protocol in detail, and investigate how the nodes interact with each other. Communication between intermediate nodes is then achieved through packet exchanges. It permits to gather data regarding the innovative rate that each node can supply. Based on this information, the nodes decide to become $\overline{N C}$ or to stay $N C$. The target of our system is to provide incentives for nodes to become $N C$ nodes and thus to improve the system performance.

\subsection{Design of Proposed Protocol}

We first consider that each node has an available upload bandwidth that is equally divided to its children nodes. Specifically, let $B_{j}^{u}$ denotes the available upload bandwidth of node $j$. If node $j$ has $N_{C_{j}}$ children nodes, then each of node $j$ 's children nodes receives $B_{j}^{u} / N_{C_{j}}$ from node $j$. Then, at the beginning, the communication protocol imposes a maximum number of download connections $L_{j}$ to node $j \in V_{i}$.

The intermediate nodes compete for the available resources (link capacities) to maximize their own multimedia quality. They can change their status from $N C$ to $\overline{N C}$ whenever they do not receive enough packets. In such cases, the nodes are awarded with an extra link connection which can be chosen by their available pool of parent nodes (the network should remain directed acyclic). Then the link rates of the new parent nodes change to grant the new network coding node with extra incoming rate. The considered game is illustrated in Fig. 1 where node 4 does not receive the desired innovative rate and it becomes NC node. This way it can be connected with node 3 to obtain the missing innovative rate. The outgoing rate of node 3 is equally split into the outgoing links. It should be noted that the nodes have a limit on the cumulative incoming and outgoing rates as well as a maximum number of connections that they can support.

\subsection{Formulation of Interactions as Congestion Games}

As discussed in the previous section, each intermediate node $j \in V_{i}$ has maximum number of links $L_{j}$ which is limited by each nodes ability for download from its parent nodes. A set of parent nodes of 
node $j$ is denoted by $P_{j}$ and is expressed as

$$
P_{j}=\left\{v_{1}, \ldots, v_{L_{j}}\right\} \subseteq\left\{V_{i} \cup V_{s}\right\}
$$

where $v_{l}, 1 \leq l \leq L_{j}$, is a node selected by node $j$, that provides download bandwidth to peer $j$. A subset of $P_{j}$ is denoted by $\mathcal{P}_{j}$. Since each node $j$ can be a network coding node $(N C)$ or a simple forwarding node $(\overline{N C})$, a set of actions (action space) of node $j$ can be expressed as

$$
\mathbf{A}_{j}=\{N C, \overline{N C}\} \times \mathcal{P}_{j} .
$$

Each action $a_{j} \in \mathbf{A}_{j}$ of node $j$ thus implies whether the node $j$ is a network coding node or not and the set of parent nodes that provide the resource to node $j$. If node $j$ decides to become $N C$, it is allowed to have extra download links. Thus, the node $j$ implementing network coding can have $L_{j}^{\prime}\left(>L_{j}\right)$ download links, which leads the node $j$ to have enlarged action space.

We assume that the nodes select their actions sequentially. This is because even if nodes are allowed to choose their actions simultaneously, it is quite rare for multiple nodes to make their decisions simultaneously. The achieved multimedia quality of a node is determined based on the download rates from its parent nodes and the number of innovative packets, as higher download rates and more innovative packets enable the node to decode the multimedia stream successfully with a higher probability. Thus, each node can maximize its achieved multimedia by selecting parents that can provide more innovative packets with higher download rates. Consequently, the utility of node $j \in V_{i} \cup V_{c}$ can be defined as the achieved quality that is determined by an selected action $a_{j}$, i.e., $U_{j}\left(a_{j}\right)$. Thus, an optimal action of node $j$ can be determined as

$$
a_{j}^{*}=\arg \max _{a_{j} \in \mathbf{A}_{j}}\left\{U_{j}\left(a_{j}\right)\right\}
$$

Note that the interactions of the nodes can be modeled as a congestion game, because the resources of each node are equally allocated only to the associated children nodes [9]. Hence, a pure strategy Nash equilibrium is always guaranteed. This will be quantified and verified based on simulation results in Section 4.

Finally, the dynamics of the system may impose periodic update of the connections for the intermediate and client nodes. Whenever a node does not receive the requested quality it drops the link that provides him with the smallest innovative rate. Then, it selects as parent the node with the highest innovative rate. It should be noted that the available set of parental nodes contains all nodes but the sources to avoid pathological cases where all nodes select the servers as their parents. After the selection of a node as a new parent, the network graph is checked to avoid cycles.

\section{SIMULATION RESULTS}

We analyze the performance of the proposed protocol for video streaming in overlay mesh networks. The transmitted video is the Foreman sequence in CIF format encoded by the scalable extension (SVC) of the latest video compression standard H.264 [10] into three quality layers. The GOP size is set to 30 frames and the frame rate is $30 \mathrm{fps}$. In each GOP the number of packets per layer is respectively $a_{1}=38, a_{2}=15$, and $a_{3}=20$. We use packets of 1500 bytes which are augmented by the TCP/IP and the network coding headers.

The overlay networks are variants of regular networks with fixed nodes per stage. Initially, all peers are linked with the same number of ancestor and descendant nodes and all network paths connecting
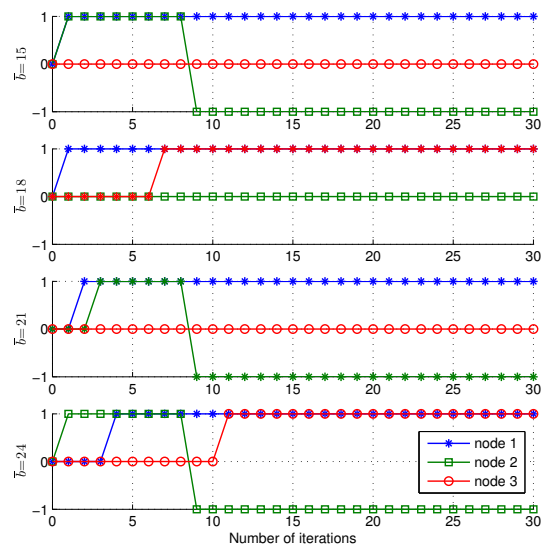

Fig. 2. Convergence process of proposed protocol for three nodes randomly selected from a random topology generated by regular networks with six stages and four nodes per stage.

servers and end users have equal distance length (in hops). The irregular topologies are generated by random link pruning and addition in the regular topologies. Pruning simply consists in removing a link from the regular topology. We further add links in the network that are not necessarily connecting nodes placed into consecutive stages. After pruning and addition operations we make sure that cycles are avoided. The link pruning probability follows uniform distribution, while link addition probability depends on the stage each node belongs to in the regular topology. Link addition probability linearly decreases with the distance from the end users.

We first examine the transmission of the Foreman CIF sequence in overlay networks consisting of six stages with four nodes per stage. The pruning and addition probabilities are set to $34 \%$, while the link's capacity $\bar{b}$ are varied in the range of $[15,24]$ packets per link. Each link experiences a $5 \%$ uniform packet loss rate. First, we study the convergence of the proposed protocol. Results for three nodes that have been randomly selected in the network are depicted in Fig. 2 for four different link capacities $\bar{b}$, namely 15, 18, 21 and 24 packets per link. The action 0 stands for $\overline{N C}$ intermediate nodes while 1 and -1 are respectively different states of a $N C$ node. When a transition from +1 to -1 (or vice-versa) happens when the node changes one of its parental nodes as it does not receive adequate innovative flow. From the results, it is obvious that in all cases the convergence is achieved after a few iterations. Therefore, our algorithm guarantees an equilibrium as discussed in Section 3.

For the sake of completeness, we present PSNR results of the proposed scheme for the transmission of the Foreman CIF sequence over an overlay network with the same settings as before. The results are illustrated in Fig. 3(a) for capacities $\bar{b}$ equal to 15, 18, 21 and 24 packets per link, respectively. From the results, we can observe that the average PSNR $(\overline{P S N R})$ increases by $0.5 \mathrm{~dB}$ when the bandwidth is sufficient. When the resources become smaller, the average quality decreases since all the clients cannot be served with the quality they requested. The distribution of resources and hence quality becomes more unbalanced. This behavior is confirmed by observing the decoding probabilities of the first and second class packets in Figs. 3 (b) and (c), respectively. As the actions taken by nodes are stable (i.e., the algorithm converges to its equilibrium) the decoding probability of the second class increases, while the decoding probability of the first class decreases. The number of nodes that are able to decode the second class and to benefit from higher quality 

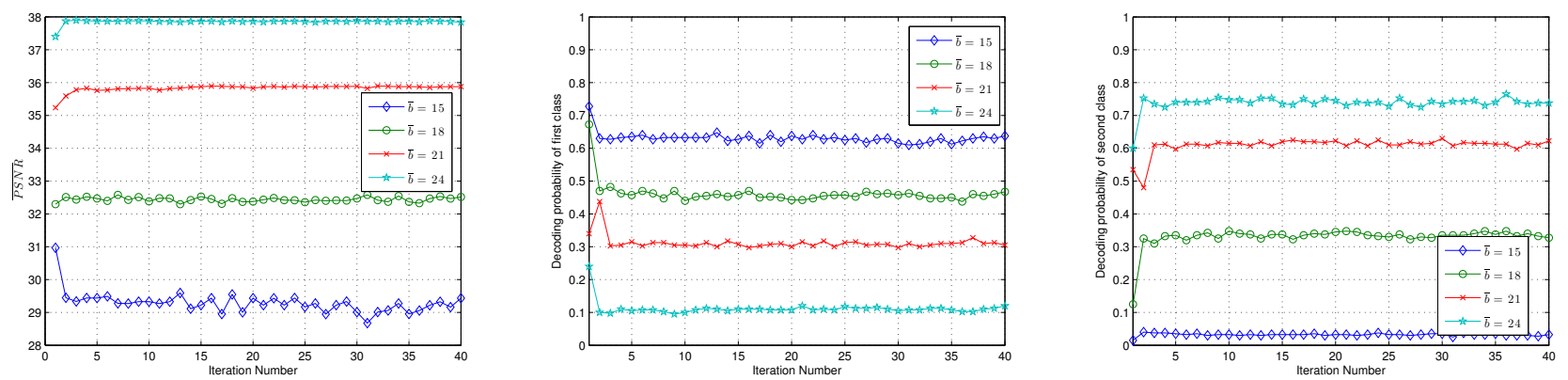

Fig. 3. Evolution of (a) decoded $\overline{P S N R}$ values, (b) decoding probability of first class and (c) decoding probability of second class with the number of iterations. Irregular networks with six stages and four nodes per stage are considered.

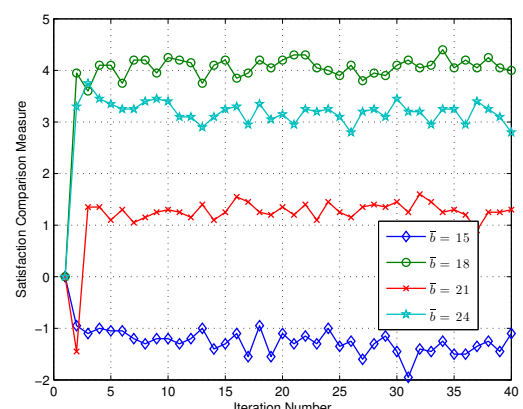

Fig. 4. Satisfaction comparison measure of the proposed protocol.

increases, which confirms the benefit of network coding and the performance of the proposed algorithm. Such a behavior is typical for a non-cooperative game as the one proposed in this paper.

Finally, we quantify the satisfaction of each client achieved based on the proposed protocol. For this, we introduce "satisfaction comparison measure" ( $\mathrm{SCM}$ ), defined as $S C M=y-x$, where $x$ denotes an initial decodable layer and $y$ is a decodable layer at equilibrium in the end of the game. For example, in the case where the video quality stays unaltered, $S C M=0$. We evaluate SCM for networks generated with the process described above and for the same settings. The average cumulative SCM $(\overline{S C M})$ for all clients are shown in Fig. 4. It is clear that nodes' satisfaction decreases (decreasing $\overline{S C M}$ ) as the game is played if the bandwidth is low. This is because the available bandwidth is not enough and the nodes are sharing the limited bandwidth. This eventually leads more nodes to decode lower layers. However, for higher available bandwidth, as the game is played, more users can share the available bandwidth, which leads them to decode higher layers on average. Therefore, the higher $\overline{S C M}$ can be achieved. The amount of $\overline{S C M}$ increment depends on the initial resources i.e. if the bandwidth is not fairly split and the available resources are such that the nodes can exploit them in a more social optimal way as for $\bar{b}=18$ larger gains in $\overline{S C M}$ are noticed.

\section{DISCUSSION}

We have presented an RNC based streaming system for multimedia transmission in overlay mesh networks. In our system, the network codes can be RNC nodes or follow "store and forward" approach. The nodes autonomously decide whether should become RNC nodes or stay "store and forward" based on the received innovative rate and the multimedia quality. Nodes becoming RNC are awarded with an extra connection and permitted to change their parental nodes. The experimental results have shown that the proposed protocol converges to a Nash equilibrium with a stable quality of service. From the experimental evaluation, it has been observed that the proposed game converges in a finite number of iterations. Future work thus may include more theoretical investigation of the convergence rate, which will be critical for the practical deployment of the algorithm in real systems.

\section{REFERENCES}

[1] M. J. Osborne and A. Rubinstein, A Course in Game Theory. The MIT Press, 1994.

[2] R. Ahlswede, N. Cai, S.-Y. R. Li, and R. W. Yeung, "Network Information Flow," IEEE Trans. Info. Theory, vol. 46, no. 4, pp. 1204-1216, Jul. 2000.

[3] N. Thomos and P. Frossard, "Raptor Network Video Coding," in Proc. ACM Int. Workshop on Mobile video, Sep. 2007.

[4] P. A. Chou, Y. Wu, and K. Jain, "Practical Network Coding," in Proc. Allerton Conf. on Commun. Control and Comput., Oct. 2003.

[5] M. Wang and B. Li, " $R$ ': Random Rush with Random Network Coding in Live Peer-to-Peer Streaming," IEEE J. Sel. Areas in Commun., vol. 25, no. 9, pp. 1655-1666, Dec. 2007.

[6] T. Ho, M. Medard, J. Shi, M. Effros, and D. R. Karger, "On Randomized Network Coding," in Proc. Allerton Annual Conf. Commun., Control, and Comput., Oct 2003.

[7] M. Wu, S. Karande, and H. Radha, "Network Embedded FEC for Optimum Throughput of Multicast Packet Video," EURASIP J. Applied Signal Process., vol. 20, no. 8, pp. 728742, Sep. 2005.

[8] N. Thomos, J. Chakareski, and P. Frossard, "Randomized network coding for UEP video delivery in overlay networks," in Int. Conf. Multimedia and Expo 2009, June 2009.

[9] H. Park and M. van der Schaar, "Quality-based Resource Brokerage for Autonomous Networked Multimedia Applications," IEEE Trans. Circuits and Syst. Video Technol., vol. 19, no. 12, pp. 1781-1792, Dec. 2009.

[10] ITU-T and ISO/IEC JTC 1, "Advanced video coding for generic audiovisual services, amendment 3: Scalable video coding," Draft ITU-T Recommendation H.264 - ISO/IEC 14496-10(AVC), April 2005. 